\title{
Automatic Choice of Denoising Parameter in Perona-Malik Model
}

\author{
A.V. Nasonov ${ }^{1}$, N.V. Mamaev ${ }^{1}$, O.S. Volodina ${ }^{1}$, A.S. Krylov ${ }^{1}$ \\ nasonov@cs.msu.ru | mamaev.nikolay93@mail.ru | olya.volodina@gmail.com | kryl@cs.msu.ru \\ ${ }^{1}$ Faculty of Computational Mathematics and Cybernetics \\ Lomonosov Moscow State University \\ Moscow, Russia
}

In this work, we propose a no-reference method for automatic choice of the parameters of Perona-Malik image diffusion algorithm for the problem of image denoising. The idea of the approach it to analyze and quantify the presence of structures in the difference image between the noisy image and the processed image as the mutual information value. We apply the proposed method to photographic images and to retinal images with modeled Gaussian noise with different parameters and analyze the effects of no-reference parameter choice compared to the optimal results. The proposed algorithm shows the effectiveness of no-reference parameter choice for the problem of image denoising.

Keywords: Image denoising, non-linear diffusion, mutual information, automatic parameter choice

\section{Introduction}

One of the main challenges in image processing is denoising, as images are often corrupted by noise during acquisition, transmission or storage. The goal is to restore the original image by removing all noise while preserving the contents. Image denoising is usually needed as a preparation step in other image processing methods. There has been a great research effort in that field, yet the problem remains unsolved. In this paper, we will use non-linear diffusion method proposed in [1] by Perona and Malik, which represents a filtered image as a solution of nonlinear diffusion equation with the original image as initial state and homogeneous Neumann boundary conditions. By choosing the diffusion parameter, one can manage to clean flat areas and preserve edges. Non-linear diffusion is an iterative process so there is a problem of stop mechanism.

Most algorithms depend on noise level and thus must be controlled by parameters entered by a user or estimated automatically. A common approach for automatic choice of the parameters is to estimate the noise level and then choose the parameters according to this noise level [2].

A less common approach is to analyze the preservation of image contents after image restoration and to pose the stopping criterion of anisotropic diffusion. For example, the work [3] analyzes the edge characteristics, the work [4] calculates image statistics for speckle noise reduction. In [5], a analysis of the contents in the difference image between the original noisy image and the processed image is performed. Its idea comes from an assumption, that in the ideal case the difference image must contain just random values without any structures from the original image. If there are structures from the original noisy image, then we have wiped out the important information as well as the noise.

In this work, we investigate the automatic choice of the parameters for Perona-Malik image diffusion for Gaussian noise for photographic and retinal images.

\section{Perona-Malik image diffusion}

One of the methods for image denoising is based on non-linear diffusion that considers the cleaned image as the solution of the heat conduction. The diffusion coefficient is chosen to reduce the diffusivity in locations, which have more likelihood to be edges. Such methods allow to preserve edges while denoising due to the right choice of coefficient. Koenderink [6] and Hummel [7] pointed out that an imaged convolved with Gaussian kernel can be viewed as the solution of the heat conduction equation with original image as initial condition.

$$
\begin{gathered}
\frac{\partial u}{\partial t}=\operatorname{div}(c \nabla u), \quad(x, t) \in \Omega \times[0, T], \\
u(x, 0)=l_{0}, \quad x \in \Omega, \\
\frac{\partial u}{\partial \vec{n}}=0, \quad(x, t) \in \partial \Omega \times[0, T],
\end{gathered}
$$

where $l_{0}$ is the input image defined in spatial domain $\Omega, c$ is the diffusion coefficient, $u(x, T)$ is the result of heat distribution at moment $T$.

In linear diffusion, the coefficient $c$ is considered to be constant and independent of the image. In nonlinear diffusion, the coefficient $c$ is a function of image gradient magnitude $c=c(|\nabla u|)$, which controls the blurring effect. Setting $c$ to 1 in interior of each region and 0 at the boundaries will encourage smoothing within a region and stop it on the edge, so that the boundaries remain sharp. In [1] Perona and Malik proposed two functions as edge-estimator:

and

$$
c_{1}(s)=\exp \left(-\left(\frac{s}{K}\right)^{2}\right)
$$

$$
c_{2}(s)=\frac{1}{1+\left(\frac{s}{K}\right)^{2}},
$$

where $K$ is the parameter of the method.

The diffusion equation can be solved numerically by simple step algorithm:

$$
\begin{gathered}
u_{n+1}=u_{n}+t_{n} \cdot c(|\nabla u|) \Delta u, \\
u_{0}=u(x, 0)=I_{0}, \\
\sum_{n} t_{n}=T .
\end{gathered}
$$


In our work, we use the model (2).

\section{Target images}

We have analyzed the automatic choice of the parameters for the Perona-Malik image diffusion algorithm for images of the following two classes:

- Photographic images from TID database [8];

- Retinal images from DRIVE database [9].

An example of those images is shown in Fig. 1.

In order to model noisy images, we have added white Gaussian noise with different levels $\sigma$ in $[1,32]$ range to the reference images.

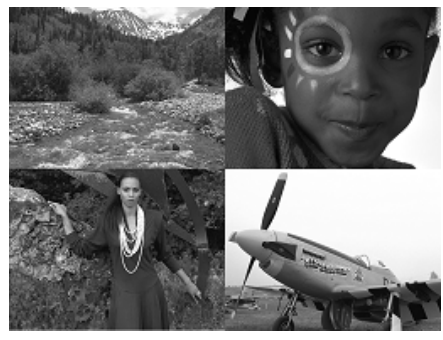

TID database [8]

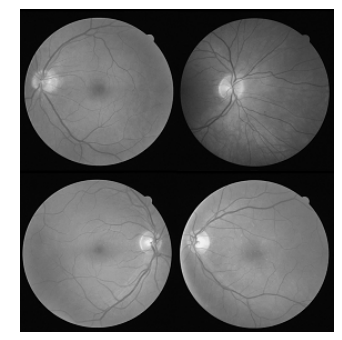

DRIVE database [9].
Fig. 1. An example of reference images used for the analysis in the paper.

\section{Full-reference parameter analysis}

For each noisy image, we have obtained a pair of $(K, T)$ parameters that maximizes PSNR and SSIM [10] metric values. We have found that for each image there is a set of $(K, T)$ values producing the results that are almost indistinguishable from the optimal result. The set is banana-shaped and lies perpendicular to the line passing though the zero point. Fig. 2 shows an example of optimal $(K, T)$ values for one of the images for different noise levels.



Noise $=3$

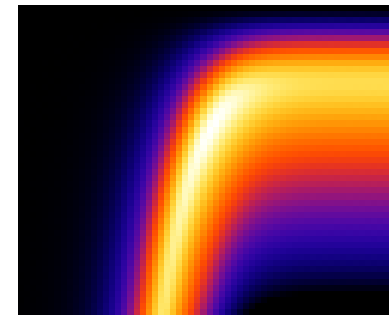

Noise $=8$
Fig. 2. A visualization of optimal $(K, T)$ parameters for an image with different noise levels in terms of PSNR. The horizontal axis represents $K$ value in logarithmic scale. The vertical axis represents $T$ value. Top-left corner is $(0,0)$ point. White regions corresponds to $(K, T)$ values that produce images with PSNR values close to the optimal value. Black regions correspond to PSNR values equal or less than PSNR for the unprocessed image.
We have also noticed that for each noise level the ratio $K / T$ can be fixed, and the parameter optimization becomes one-dimensional, but for different noise level the optimal ratio $K / T$ set is different.

In order to go from two-dimensional to onedimensional parameter optimization for any noise level, we have analyzed the behavior of optimal $(K, T)$ values and have found out that a set of optimal points $(\log K, \sqrt{T})$ lies along a line. Therefore, we introduce single-argument parameterization for $(K, T)$ values:

$$
\begin{gathered}
K=q_{1} q_{2}^{p}, \\
T=p^{2},
\end{gathered}
$$

where the coefficients $q_{1}$ and $q_{2}$ are chosen experimentally by optimizing the full-reference metrics values.

For both TID and DRIVE images, we have fixed $q_{1}=0.1$ and optimized $q_{2}$ value. The ranges of optimal values for TID images and for DRIVE images are different, but they intersects. We have chosen $q_{2}=4600$ from the intersection.

\section{No-reference parameter choice}

We use the algorithm [5] for non-reference parameter choice. The algorithm is based on the assumption that the difference between input noisy and denoised images should not have features belonging to original image. In order to detect the presence of these features, the algorithm analyses the eigenvalues of Hessian matrix for scale and direction evaluation of ridges and edges. The outcome of the algorithm is value $\mu$ - the mutual information that can be expressed as the structure-to-noise ratio for the difference image. The lower the value $\mu$ is, the less details are corrupted compared to noise removal.

We use the following scenario: an image denoising algorithm is executed with different parameters, then the mutual information $\mu$ value is calculated between the input image and each denoising result, and the image that minimizes the mutual information is chosen as the optimal result. In practice, there can be several local minima, and a special analysis should be performed in order to choose the optimal result.

After replacing the two-parameter model with the single-parameter model (3), we find the optimal $p$ value using both full-reference and no-reference approach based on calculating the mutual information coefficient.

It has been found that mutual information correlates well with PSNR and SSIM values for noise level $\sigma>2$. An example is shown in Fig. 3. A argument where PSNR and/or SSIM reaches its maximum is close to a local minimum of $\mu(p)$ function. In the case of several local minima points, we find the one that maximizes the drop:

$$
p_{\text {opt }}=\arg _{p} \max _{p^{\prime}<p} \mu\left(p^{\prime}\right)-\mu(p) .
$$

In the case of very low noise level $(\sigma \leq 2)$, the method has limited application. Non-linear diffusion improves the image very little in the case of low noise. 
The difference image has low magnitude, so the mutual information coefficient is low, and local minimum point becomes unstable or even disappears.

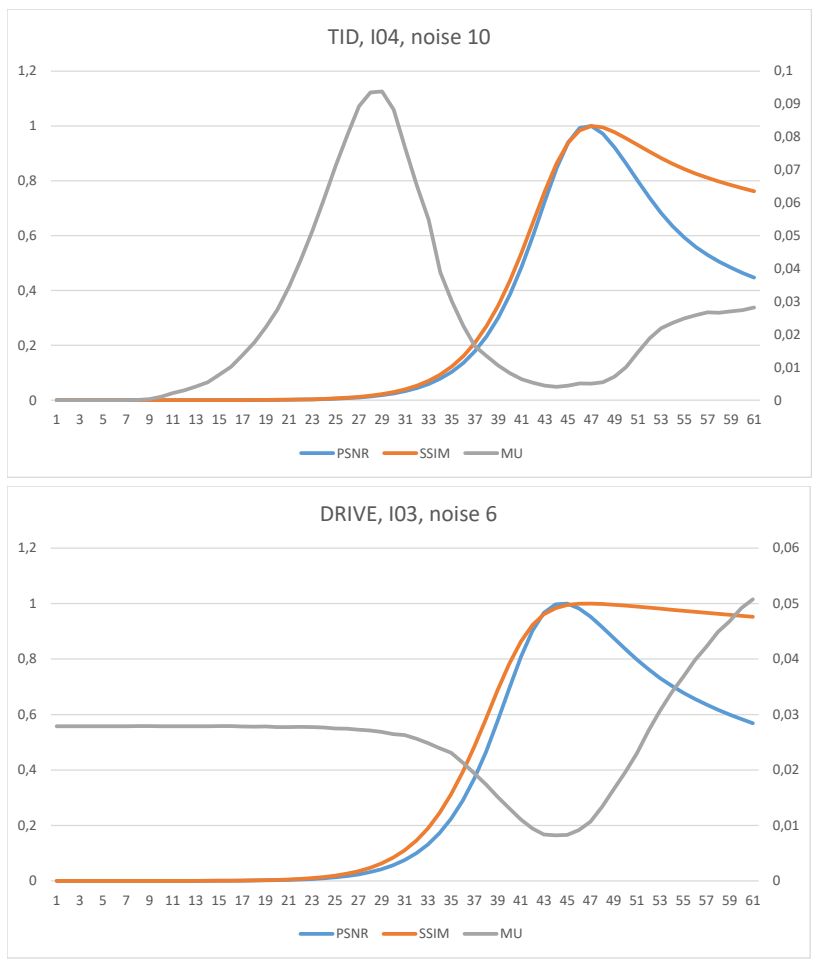

Fig. 3. Examples of the dependence of PSNR, SSIM and mutual information on the parameter $p$ corresponding to denoising strength. The PSNR and SSIM values are normalized into $[0,1]$ range.

\section{Results}

The numerical results for different scenarios of denoising parameter choice are presented in table 1. The results are averaged for all the images with noise level $\sigma>2$.

Despite the fact that the proposed no-reference algorithm has worse PSNR and SSIM values than the optimal ones, the difference between the results of the proposed algorithm and the optimal results is practically indistinguishable, and the effectiveness of image denoising is clearly visible.

The individual results are shown in Fig. 4, 5, 6.

\section{Conclusion}

The paper has shown that the parameters of the Perona-Malik image denoising algorithm can be automatically and effectively chosen by the algorithm that analyzes the presence of structures from the input image in the difference image.

The work was supported by Russian Science Foundation grant 17-11-01279.

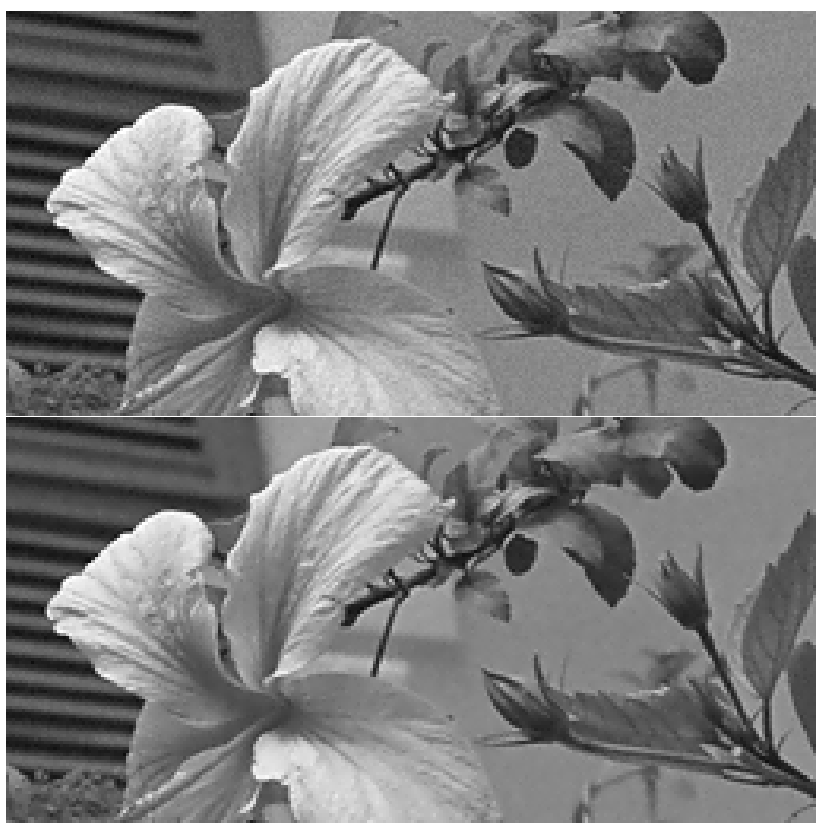

Fig. 4. Denoising by the proposed method. TID image I07, noise $\sigma=4$.

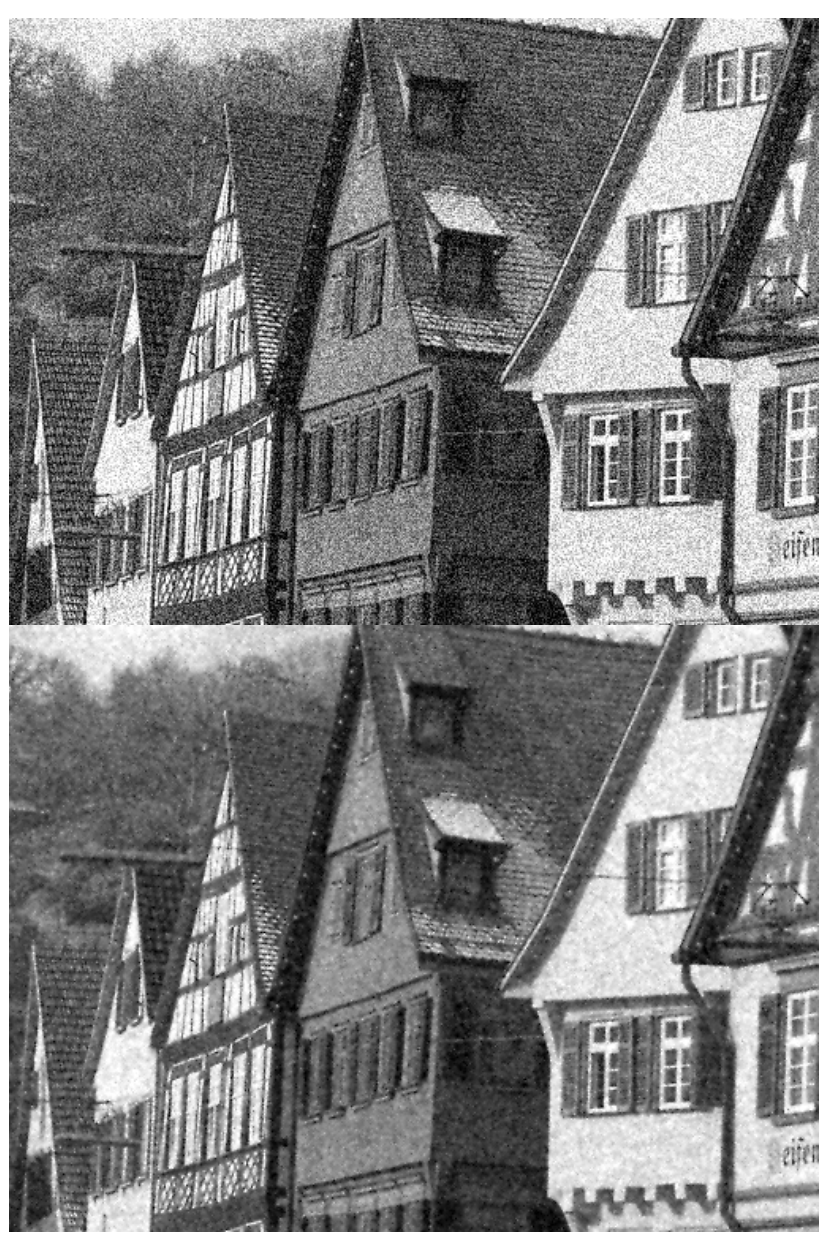

Fig. 5. Denoising by the proposed method. TID image I08, noise $\sigma=32$. 


\begin{tabular}{|l|c|c|c|c|}
\hline \multirow{2}{*}{ Optimization method } & \multicolumn{2}{|c|}{ TID } & \multicolumn{2}{c|}{ DRIVE } \\
\cline { 2 - 5 } & PSNR & SSIM & PSNR & SSIM \\
\hline Input noisy images & 30.76 & 0.8083 & 30.76 & 0.6174 \\
Full-reference, double-parameter, by PSNR & 34.25 & 0.9252 & 39.21 & 0.9293 \\
Full-reference, double-parameter, by SSIM & 34.10 & 0.9286 & 38.87 & 0.9338 \\
Full-reference, single-parameter, by PSNR & 34.22 & 0.9236 & 38.99 & 0.9224 \\
Full-reference, single-parameter, by SSIM & 34.03 & 0.9267 & 38.40 & 0.9297 \\
No-reference, single-parameter, by MU (proposed) & 33.77 & 0.9135 & 38.97 & 0.9218 \\
\hline
\end{tabular}

Table 1. PSNR and SSIM results for different scenarios of denoising parameter choice for TID and DRIVE images.

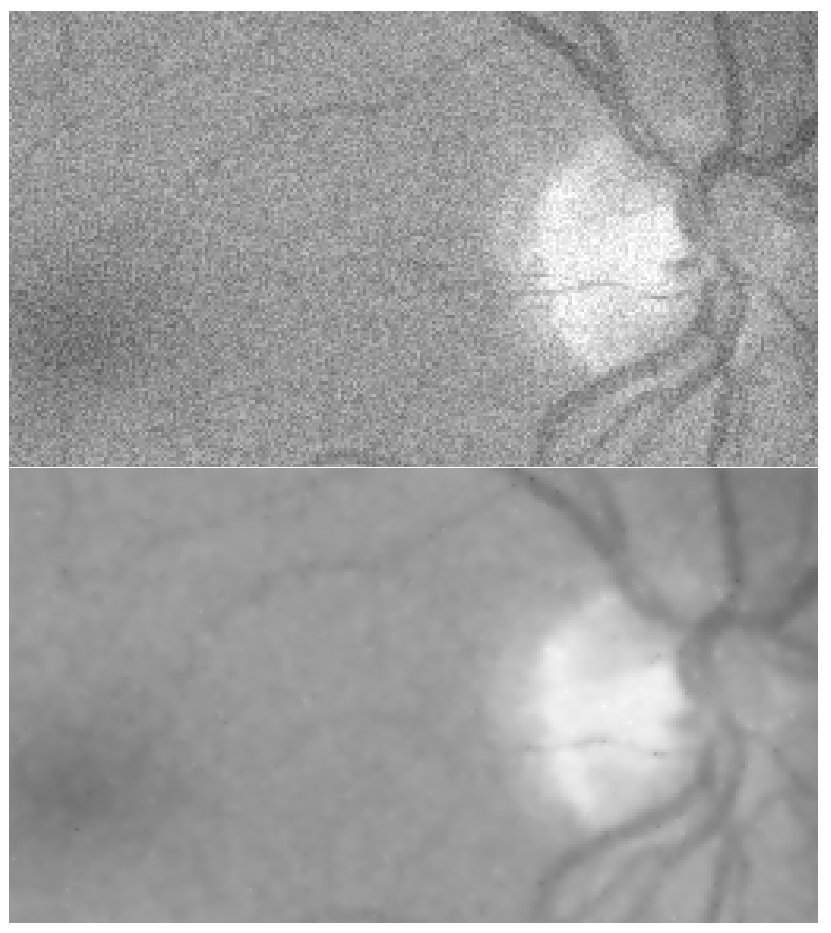

Fig. 6. Denoising by the proposed method. DRIVE image I02, noise $\sigma=13$.

\section{References}

[1] Pietro Perona and Jitendra Malik. Scale-space and edge detection using anisotropic diffusion. IEEE Transactions on pattern analysis and machine intelligence, 12(7):629-639, 1990.

[2] Karl Krissian and Santiago Aja-Fernández. Noise-driven anisotropic diffusion filtering of mri. IEEE transactions on image processing, 18(10): 2265-2274, 2009.

[3] Chourmouzios Tsiotsios and Maria Petrou. On the choice of the parameters for anisotropic diffusion in image processing. Pattern recognition, 46(5):1369-1381, 2013.

[4] Santiago Aja-Fernández and Carlos AlberolaLópez. On the estimation of the coefficient of variation for anisotropic diffusion speckle filtering. IEEE Transactions on Image Processing, 15 (9):2694-2701, 2006.
[5] Nikolay Mamaev, Dmitry Yurin, and Andrey Krylov. Choice of the parameter for bm3d denoising algorithm using no-reference metric. In 2018 rth European Workshop on Visual Information Processing (EUVIP), pages 1-6. IEEE, 2018.

[6] Jan J Koenderink. The structure of images. $B i$ ological cybernetics, 50(5):363-370, 1984.

[7] Robert A Hummel. Representations based on zero-crossings in scale-space. In Readings in Computer Vision, pages 753-758. Elsevier, 1987.

[8] N. Ponomarenko, L. Jin, O. Ieremeiev, V. Lukin, K. Egiazarian, J. Astola, B. Vozel, K. Chehdi, M. Carli, F. Battisti, and C.-C. Jay Kuo. Image database tid2013: Peculiarities, results and perspectives. Signal Processing: Image Communication, 30:57-77, 2015.

[9] M.D. Abramoff J.J. Staa and, M. Niemeijer, M.A. Viergever, and B. van Ginneken. Ridge based vessel segmentation in color images of the retina. IEEE Transactions on Medical Imaging, 23:501-509, 2004.

[10] Z. Wang, A. Bovik, H. Sheikh, and E. Simoncelli. Image quality assessment: from error visibility to structural similarity. IEEE Transactions on Image Processing, 13(4):600-612, 2004. 